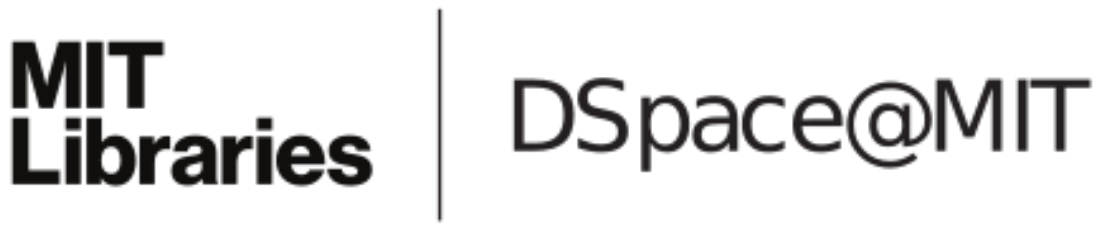

\author{
MIT Open Access Articles
}

\section{Dynamics and trajectory optimization for a soft spatial fluidic elastomer manipulator}

The MIT Faculty has made this article openly available. Please share how this access benefits you. Your story matters.

Citation: Marchese, Andrew D., Russ Tedrake, and Daniela Rus. “Dynamics and Trajectory Optimization for a Soft Spatial Fluidic Elastomer Manipulator." 2015 IEEE International Conference on Robotics and Automation (ICRA) (May 2015).

As Published: http://dx.doi.org/10.1109/ICRA.2015.7139538

Publisher: Institute of Electrical and Electronics Engineers (IEEE)

Persistent URL: http://hdl.handle.net/1721.1/101035

Version: Author's final manuscript: final author's manuscript post peer review, without publisher's formatting or copy editing

Terms of use: Creative Commons Attribution-Noncommercial-Share Alike 


\title{
Dynamics and Trajectory Optimization for a Soft Spatial Fluidic Elastomer Manipulator
}

\author{
Andrew D. Marchese, Russ Tedrake, and Daniela Rus
}

\begin{abstract}
The goal of this work is to develop a soft robotic manipulation system that is capable of autonomous, dynamic, and safe interactions with humans and its environment. First, we develop a dynamic model for a multi-body fluidic elastomer manipulator that is composed entirely from soft rubber and subject to the self-loading effects of gravity. Then, we present a strategy for independently identifying all unknown components of the system: the soft manipulator, its distributed fluidic elastomer actuators, as well as drive cylinders that supply fluid energy. Next, using this model and trajectory optimization techniques we find locally optimal open-loop policies that allow the system to perform dynamic maneuvers we call grabs. In 37 experimental trials with a physical prototype, we successfully perform a grab $92 \%$ of the time. By studying such an extreme example of a soft robot, we can begin to solve hard problems inhibiting the mainstream use of soft machines.
\end{abstract}

\section{INTRODUCTION}

Industrial-style manipulators have discrete joints and rigid links. They have been transformative for industrial repetitive tasks. However, these robots are often considered too rigid for human-centered environments where the tasks are unpredictable and the robots have to ensure that their interaction with the environment and with humans is safe. Our goal is to develop soft robot manipulators capable of autonomous, safe, and dynamic interactions with people and their environments. In this paper we present a suite of algorithms for dynamically controlling a soft fluidic elastomer manipulator acting under gravity.

In this work we provide an approach for dynamically controlling soft robots. That is, an entirely soft fluid-powered multi-segment robot can be autonomously positioned to accomplish tasks outside of its gravity compensation envelope. Specifically, we begin by developing a dynamic model for such a soft manipulation system as well as a computational strategy for identifying the model. Then, we use this model and trajectory optimization methods to execute dynamic motion plans. Through simulation and experiments we demonstrate repeatable positioning of the aforementioned manipulator to states outside of the statically reachable workspace in dynamic maneuvers we call grabs (See Fig. 1). For example, consider a soft manipulator that can safely and dynamically interact with humans by quickly grabbing objects directly from a human's hand. To the best of our knowledge, this is the first instance of dynamic motion control for a soft fluidic elastomer robot.

Andrew D. Marchese, Russ Tedrake, and Daniela Rus are with the Computer Science and Artificial Intelligence Laboratory, Massachusetts Institute of Technology, 32 Vassar St. Cambridge, MA 02139, USA, \{andy, russt, rus\}@csail.mit.edu
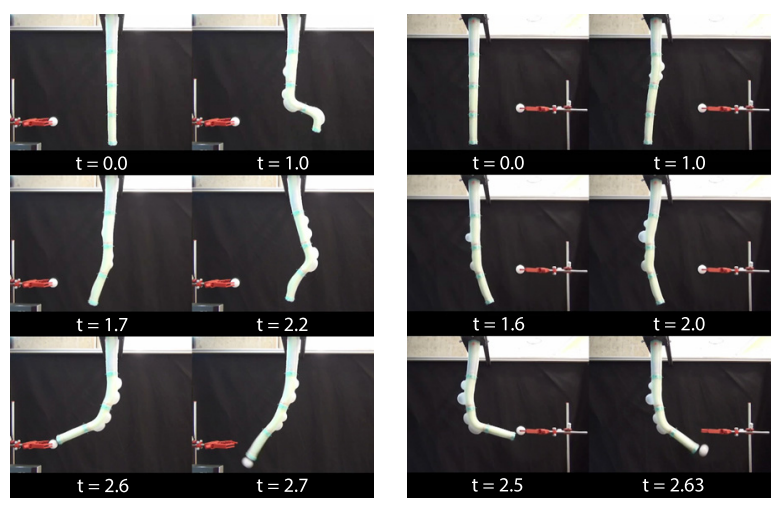

Fig. 1. Sequenced photographs from experiments three and four.

\section{A. Prior Work}

Soft robots have continuously deformable backbones that undergo large deformations. This attribute means soft robots are a subclass of continuum robots, as reviewed by Robinson and Davies [1]. However, not all continuum robots are soft and even continuum robots referred to as soft have varying degrees of rigidity.

1) Dynamics and Control for Continuum Robots: Purely kinematic approaches to continuum robot control and planning work in simulation and when the robot is sufficiently constrained by the rigidity of its actuators or backbone. For example, Hannan and Walker [2] develop novel continuum kinematics for a hyper-redundant elephant trunk and demonstrate how these enable capabilities like obstacle avoidance. Jones and Walker [3] [4] provide kinematic algorithms for controlling the shape of multi-segment continuum manipulators. Chirikjian and Burdick [5] use a continuous backbone model to plan optimal hyper-redundant manipulator configurations using calculus of variations. Additionally, Xiao and Vatcha [6] introduce a planar continuum arm planner that enables simulated grasping in uncertain, cluttered environment.

Dynamic models of continuum robots open the door for a variety of control techniques. Chirikjian [7] uses a continuum approach to model the dynamics of a hard hyper-redundant manipulator and uses this for computed torque control. Gravagne and Walker [8] dynamically model the Clemson Tentacle Manipulator, a hard continuum robot, and show a PD plus feed-forward regulator is sufficient for stabilizing the system. They further develop a large deflection model and controller in [9]. Snyder and Wilson [10] [11] dynamically model polymeric pneumatic tubes subject to tip loading using a bending beam model but do not use this for control. Using a Lagrangian approach Tatlicioglu, Walker, and Dawson [12] 
develop a dynamic model for and provide simulations of a planar extensible continuum manipulator. Braganza, Dawson, Walker, and Nath [13] develop a neural network controller for continuum robots such as OctArm [14] based on a dynamic model.

2) Dynamics and Control for Soft Elastomer Robots: To the best of our knowledge, highly compliant robots whose bodies are made from soft elastomer and distributed fluidic actuators have not used dynamic model-based control. Prior work in this field uses model-free open-loop control policies, but because this existing work does not derive control policies from nonlinear dynamic models these approaches cannot efficiently plan motions for novel tasks without sufficient trial-and-error. Most fluid powered soft robots use modelfree open-loop valve sequencing to control body segment bending. That is, a control valve is turned on for a userdetermined duration of time to pressurize an elastomer actuator and then off to either hold or deflate the actuator [15], [16], [17], [18], [19]. Previously we have demonstrated an approach to motion control for planar soft elastomer manipulators using closed-loop kinematic control [20], [21], but again a dynamic model was not used in the control strategy. Open-loop model-free control is also common for soft elastomer robots that do not use pneumatic actuation [22], [23], [24], [25]. Luo, Agheli, and Onal [26] develop and verify a planar dynamic model for a soft snake but do not use it for control. Martinez et al. [27] develop manually operated elastomer tentacles containing 9 PneuNet actuators embedded within 3 body segments.

\section{B. Contributions}

Our work builds on this previous work. Specifically, this paper contributes the following:

- A dynamic model for a fluid powered manipulator made entirely from soft elastomer as well as a process for fitting this model to experimental data;

- Dynamic control algorithms that allow such a soft manipulator operating under gravity to be precisely positioned;

- A manipulation primitive built on these dynamic control algorithms, grabbing;

- Extensive experiments with a physical prototype.

\section{DEVICE OVERVIEW}

To start, we provide the reader with a brief overview of the soft arm prototype and its drive mechanisms developed by the authors in [28]. The soft arm is pictured in an unactuated configuration in the left panel of Figure 2. It is composed entirely of low durometer rubber and is powered by fluidic elastomer actuators. These actuators are distributed throughout the arm's four body segments and allow each segment to bend with two actuated degrees of freedom. Driving actuation is an array of fluidic drive cylinders (Fig. 2 right). These devices consist of a fluidic cylinder at (a) coupled to an electric linear actuator at (b). They move fluid into and out of the arm's soft actuators in a closed circuit and provide continuous adjustment of fluid flow.
The actuated region of one of the manipulator's soft arm

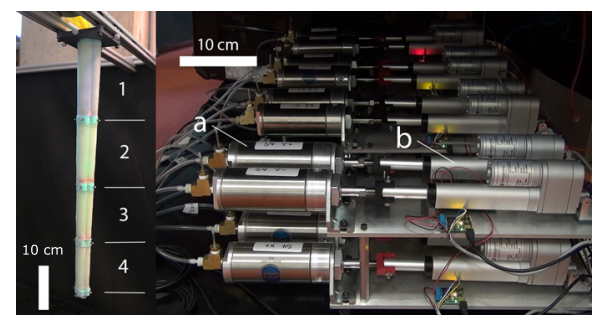

Fig. 2. Left: A soft continuum manipulator composed entirely from low durometer rubber developed by the authors in [28]. Right: An array of high capacity fluidic drive cylinders [20] used to drive the manipulator's distributed fluidic elastomer actuators.

segments is observed to bend with approximately constant curvature $\kappa$ and bend angle $\theta$ (i.e. $\kappa=\frac{\theta}{L}$ ) within a sagittal plane defined by the bend angle orientation $\gamma$. In order to transform from a segment's base to a point $s$ along the neutral axis of its actuated region, i.e. $s=[0, L]$ where $L$ is undeformed actuator length, we use the following kinematic model transformation

$$
\mathbf{S}_{s}^{\text {base }}=\mathbf{R}_{z}(\gamma) \mathbf{T}_{z}\left(L_{P}\right) \mathbf{R}_{y}\left(\frac{\kappa s}{2}\right) \mathbf{T}_{z}(d(\kappa s)) \mathbf{R}_{y}\left(\frac{\kappa s}{2}\right),
$$

where $\mathbf{R}$ and $\mathbf{T}$ are rotations and translations about and along the subscript axes and $L_{P}$ is the length of the segment's unactuated region and accounts for deadspace produced by channel inlets and/or soft end-plate connectors. This model is consistent with continuum manipulator literature [29] and is developed and validated in the context of the soft fluidic elastomer manipulator in [28].

The transformation from base to tip of a multi-segment soft arm composed of $N$ segments confined to a sagittal plane defined by $\gamma$ can be represented by cascading single segment transformations together

$$
\mathbf{M}_{\text {tip }}^{\text {base }}=\mathbf{S}_{\text {tip }}^{\text {base }}\left(\gamma, \theta_{1}\right) \mathbf{S}_{\text {tip }}^{\text {base }}\left(0, \theta_{2}\right) \cdots \mathbf{S}_{\text {tip }}^{\text {base }}\left(0, \theta_{N}\right) .
$$

\section{DYNAMIC MODEL}

To begin, we develop a dynamic model. The benefit of using a dynamic model within the iterative learning control algorithm is that control policies can be generated using a model-based open-loop policy search algorithm, such as trajectory optimization, and these are well-suited for underactuated systems.

\section{A. Energetics}

Our objective is to write the equations of motion for this soft fluidic elastomer manipulator. To do this we can first find the potential, kinetic, and input components of energy for a single arm segment and then use a Lagrangian approach to derive the equations of motion with respect to the segment's generalized coordinate. A fundamental difference between soft and hard robot manipulators is in the way energy is stored. In a soft fluidic elastomer manipulator, input fluid energy is delivered from a power supply and stored as both strain energy along its continuum segments $V_{\epsilon}$ 
and gravitational potential energy $V_{g}$. Both forms of stored energy serve to deform the manipulator and are transferred to kinetic energy $T$.

1) Potential Energy of a Segment: Consider a single arm segment deforming in a sagittal plane defined by a fixed $\gamma$. By approximating the center of mass to be located half-way along the segment's neutral axis, we can use $S_{s}^{\text {base }}$ to express the center of mass position in $\mathbb{R}^{3}$ as $\left(x_{m}(\theta), y_{m}(\theta), z_{m}(\theta)\right)$. Bend angle $\theta$ is understood to be time dependent. The gravitational potential energy of the segment is

$$
V_{g}(\theta)=m g z_{m}(\theta)
$$

where $m$ is the segment's mass and $g$ is the gravitational constant. For a fluidic soft manipulator made of deformable elastomer, a significant component of potential energy is strain energy. For strain below $60 \%$, we can approximate the stress strain relationship of the arm segment's outer layer with a constant elastic modulus $E$. This was determined from the specific material properties of the chosen elastomer. With this, the strain energy developed in an actuated channel is

$$
V_{\epsilon}=\frac{1}{2} \vee E \epsilon^{2} \rightarrow V_{\epsilon}=\frac{1}{2} \pi \bar{t}(\bar{h}+\bar{t}) L E \epsilon^{2}
$$

where $\epsilon$ is material strain, $\vee$ is the material volume incurring strain, and $\bar{t}$ and $\bar{h}$ are the wall thickness and diameter of the actuated channel. In a segment subject to circumferential and longitudinal strain that deforms under constant curvature, material strain $\epsilon$ and bend angle $\theta$ can be related by decomposing the actuated region into $J$ cross-sectional $\mathrm{x}-\mathrm{y}$ slices of z-axis length $w$ as outlined in [28] and the law of cosines

$$
\epsilon_{j}=\frac{\bar{h}_{j}}{w_{j}} \sqrt{2-2 \cos \theta_{j}} \quad \forall j=0 \ldots J \rightarrow \epsilon=\frac{\bar{h}}{w} \theta .
$$

There are several important observations that allow us to express this relationship between $\epsilon$ and $\theta$ : First, the dimensions of each slice are uniform under the aforementioned constant curvature assumption. Second, in general $\bar{h}$ is not constant, but rather changes as a function of strain $\bar{h}(\epsilon)$ and this is consistent with the analysis contained in [15] where pneumatic channels similar to the type described here increase in stiffness and potential energy when pressurized. However, we observe that after undergoing initial circumferential expansion, the diameter of the actuated channels here changes little. Approximating the diameter $\bar{h}$ to be constant is valid to describe the regime of operation after the initial circumferential change. Lastly, using the small angle approximation $\cos \theta \approx 1-\frac{\theta^{2}}{2}$ for the argument $\frac{\theta}{J}$ where $J$ is chosen such that the approximation is valid, we can linearize the relationship between $\epsilon$ and $\theta$ in order to arrive at a constant stiffness coefficient and help reduce the complexity of the model.

Now, we can write strain energy in the segment as a function of bend angle

$$
V_{\epsilon}(\theta)=\frac{1}{2}\left(\frac{\pi \bar{t}(\bar{h}+\bar{t}) L E \bar{h}^{2}}{w^{2}}\right) \theta^{2} \quad \rightarrow \quad V_{\epsilon}(\theta)=\frac{1}{2} k \theta^{2},
$$

where $k$ is an effective stiffness for the generalized coordinate $\theta$. The total potential energy of the arm segment in the sagittal plane defined by $\gamma$ is $V(\theta)=V_{g}+V_{\epsilon}$.

2) Input to a Segment: We develop an independent generalized force $\tau$ that acts on an arm segment by differentiating the total potential energy with respect to the generalized coordinate, i.e. $\tau=\frac{\partial}{\partial \theta} V$

$$
\tau=k \theta+a g L m \cos \left(\frac{\theta}{2}\right) \theta-\frac{1}{4} g L m \sin \left(\frac{\theta}{2}\right)\left(-1+a \theta^{2}\right)
$$

We can substitute in the approximations $\sin \left(\frac{\theta}{2}\right) \approx \theta$ and $\cos \left(\frac{\theta}{2}\right) \approx 1-\frac{1}{8} \theta^{2}$ with less than $5 \%$ error at $\theta$ equal to $50^{\circ}$ and $100^{\circ}$ respectively

$$
\tau=k \theta+\frac{1}{8}(1+8 a) g \operatorname{LL} \theta-\frac{1}{4} \operatorname{ag} \operatorname{Lm} \theta^{3} .
$$

This approximation will help simplify the identification process in Section IV-C. Next, we can express the change in channel volume $\mathbb{V}_{c}$ as a function of material strain and, because of our aforementioned strain assumption, a function of $\theta$

$$
\mathbb{V}_{c}=\frac{1}{2} \frac{\pi \bar{h}^{2}}{4} L \epsilon \rightarrow \mathbb{V}_{c}=\frac{\pi \bar{h}^{3} L}{8 w} \theta
$$

Substituting this into the generalized force yields:

$$
\tau=-\frac{128 a g m w^{3}}{L^{2} \pi^{3} \bar{h}^{9}} \mathbb{V}_{c}^{3}+\left(\frac{8 k w}{\pi \bar{h}^{3} L}+\frac{(1+8 a) g m w}{\pi \bar{h}^{3}}\right) \mathbb{V}_{c},
$$

revealing that there is a cubic relationship between the generalized force and the change in channel volume.

\section{B. Multi-Segment Equations of Motion}

We can write the equations of motion for a multi-segment soft manipulator using multiple generalized coordinates as follows. The center of mass position of the $n^{\text {th }}$ soft segment is represented by $\mathbf{P}_{n}$ and can be expressed as

$$
\mathbf{P}_{n}=\mathbf{M}_{\text {tip } p_{n-1}}^{\text {base }} \mathbf{S}_{\frac{L_{n}}{2}}^{\text {base }} \mathbf{0} \quad \forall n=1 \ldots N,
$$

where $\mathbf{0}$ is a vector of zeros. The total kinetic energy of a manipulator with $N$ segments is

$$
T=\sum_{n=1}^{N} \frac{1}{2} m_{n} \frac{d}{d t} \mathbf{P}_{n} \cdot \frac{d}{d t} \mathbf{P}_{n}
$$

And the total potential energy is

$$
V=\sum_{n=1}^{N} \frac{1}{2} k_{n} \theta_{n}^{2}+g \sum_{n=1}^{N} m_{n} \mathbf{P}_{n} \cdot \hat{\mathbf{k}}
$$

Using the Lagrangian $L=T-V, N$ independent nonlinear equations of motion can be written, one for each generalized coordinate

$$
\frac{d}{d t} \frac{\partial L}{\partial \dot{\theta}_{n}}-\frac{\partial L}{\partial \theta_{n}}=\tau_{n}-b_{n} \dot{\theta}_{n} \quad \forall n=1 . . N .
$$

where $b$ is a damping term used to account for the nonconservative nature of the generalized forces. The soft robot 
dynamics can now be written in traditional manipulator equation form

$$
\mathbf{H}(\boldsymbol{\theta}) \ddot{\boldsymbol{\theta}}+\mathbf{C}(\boldsymbol{\theta}, \dot{\boldsymbol{\theta}}) \dot{\boldsymbol{\theta}}+\mathbf{G}(\boldsymbol{\theta})=\mathbf{B} \tau
$$

Figure 3 provides an illustration of this model for a soft manipulator composed of four segments. The sagittal plane is defined by a traditional rotational degree of freedom $\gamma$ located at the manipulator's base. In the most general case, the dynamic model is parameterized by four generalized coordinates $\theta_{1} \ldots \theta_{4}$ and four corresponding segment masses $\mathbf{m}$, generalized stiffnesses $\mathbf{k}$, and damping coefficients $\mathbf{b}$. Additionally there are three generalized input forces $\tau$.

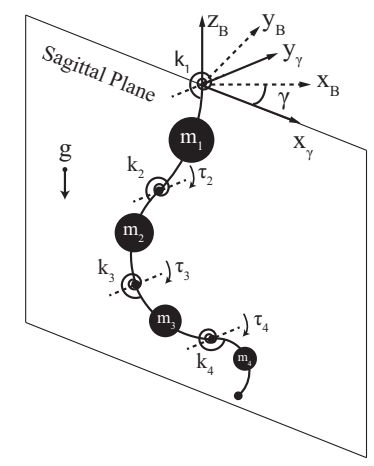

Fig. 3. Visualization of the multi-segment soft manipulator model. The first soft segment is unactuated.

\section{SYSTEM IDENTIFICATION}

In order to use the dynamic model developed in Section III for automated control we must first develop a strategy for identifying the model's unknown physical parameters. In addition to this, we must also define an approach for identifying an accurate model for the manipulator's soft actuators as well as its drive mechanisms. In this section we first present a high-level algorithm used to identify the aggregate manipulation system composed of three distinct subsystems: fluidic drive cylinders, distributed soft actuators, and the soft manipulator. Then, we look specifically at how these unknown model parameters arise from each subsystem.

\section{A. Approach Overview}

Identification of the aggregate dynamical manipulation system arm is performed by iteratively adjusting a parameter set $\mathbf{p}$ such that a model instantiated from $\mathbf{p}$ follows the same $N$-segment endpoint Cartesian trajectory as measured on the physical system. Specifically, we do this by solving the nonlinear optimization within Algorithm 1 for a locally optimal parameter set $\mathbf{p}^{*}$. Here, $\mathbf{E}_{n, i}$ is a discrete trajectory of the measured cartesian endpoint coordinates of the $n^{\text {th }}$ arm segment. The manipulator state trajectory $\mathbf{x}(t)$ is composed of segment bend angles $\boldsymbol{\theta}$ and corresponding velocities $\dot{\boldsymbol{\theta}}$. The function FORWARDKIN $n$ uses the multi-segment transformation to return the cartesian endpoint coordinates of the $n^{\text {th }}$ arm segment. The function UPDATEMODEL instantiates arm according to the parameter set $\mathbf{p}$ and the function SIMULATE forward simulates the response of the dynamic

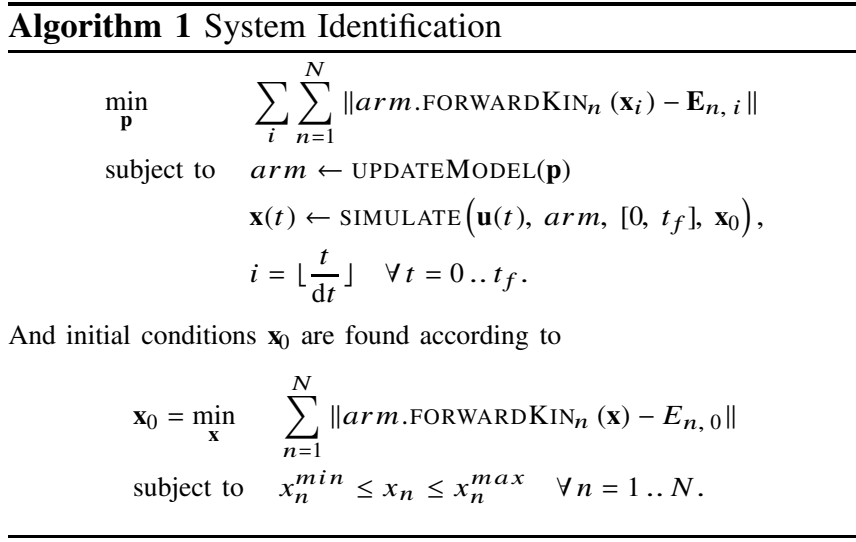

model to input trajectory $\mathbf{u}(t)$ over the time interval $t=$ $\left[0, t_{f}\right]$ from initial conditions $\mathbf{x}_{0}$.

The aggregate manipulation system arm consists of four fluidic drive cylinder pairs (Figure 2 right panel) connected to eight fluidic elastomer actuators distributed within the soft manipulator. We break this aggregate system into three distinct subsystems with the following input $\rightarrow$ output relationships:

1) Fluidic Drive Cylinders: reference inputs $\mathbf{u} \rightarrow$ cylinder displacements $\mathbb{V}_{s}$

2) Fluidic Elastomer Actuators: cylinder displacements $\mathbb{V}_{S} \rightarrow$ generalized torques $\tau$

3) Soft Manipulator: generalized torques $\tau \rightarrow$ manipulator states $\mathbf{x}$

Both the dynamic manipulator model and system identification algorithm were implemented using Drake [30], which is an open-source planning, control, and analysis toolbox for nonlinear dynamical systems.

\section{B. Fluidic Drive Cylinders}

Volumetric fluid changes to each agonistic pair of embedded channels within a soft arm segment are controlled by a pair of position-controlled fluidic drive cylinders, a device developed by the authors in [20]. In this work we further develop and identify the device's dynamic model. Each pair is identified as an independent subsystem, and under the sagittal plane assumption $N$ of these subsystems are required.

The input to each subsystem is $u$, a reference differential volumetric displacement to the position controlled cylinder pair and the output of each subsystem is $\mathbb{V}_{s}$, the differential volumetric displacement of the cylinders. One of two identical cylinders in the pair is driven at a time and pressurizes either half of the attached bending segment. To experimentally identify this subsystem we conduct several trials of the same experiment. The experiment consists of exciting the system with a reference wave $w(t)$. We fit a second order state space model to measured input-output data from one of five trials and then validated the model prediction against the remaining four trials. An example verification is shown in Figure 4. The identification and verification process was repeated for each of the 4 cylinder pairs used in later experiments. 

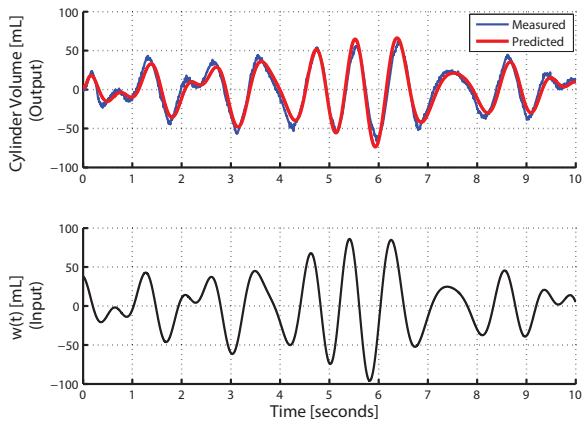

Fig. 4. Example experimental identification of a position controlled fluidic drive cylinder subsystem. The identification process consists of exciting each independent subsystem with several randomized wave profiles and fitting and verifying a two state LTI black-box model to measured input-output data. Top: model predicted and measured output in blue and red respectively. Bottom: subsystem input.

\section{Fluidic Elastomer Actuators}

To identify the dynamics of the arm's soft actuators, we rely on the predicted cubic relationship between internal channel volume $\mathbb{V}_{c}$ and generalized torque $\tau$ as developed in Section III-A.2. Also, the relationship between piston pressure $p_{s}$ and channel volume $\mathbb{V}_{c}$ indicates a delay due to the impedance of the transmission line $R_{t}$

$$
\begin{aligned}
& \dot{p}_{s}=\frac{1}{C} \dot{\mathbb{V}}_{s}(t)-\frac{1}{C R_{t}} p_{s}+\frac{1}{C_{a} C R_{t}} \mathbb{V}_{c}, \\
& \dot{\mathbb{V}}_{c}=-\frac{1}{\left(C+C_{a}\right) R_{t}} \mathbb{V}_{c}+\frac{C_{a}}{\left(C+C_{a}\right) R_{t}} p_{s}
\end{aligned}
$$

where $\mathbb{V}_{s}(t)$ is considered the subsystem input and $C_{a}$ and $C$ is a first order approximation of the actuator's channel compliance and air respectively. Combining these effects we define a simplified identifiable model in the form

$$
\tau(t)=c \mathbb{V}_{s}^{3}\left(t-t_{d}\right) .
$$

The model constants $\mathbf{c}$ for each actuator pair and a single $t_{d}$ are added to the main algorithm's parameter set $\mathbf{p}$ for identification, as the soft actuators are subject to dynamic fatigue and their performance is susceptible to change over time.

To validate this input output relationship, we again perform several trials of the aforementioned experiment, this time deriving actuator torque through a custom apparatus that measures the blocked tip force exerted by a segment fixed at its base. Figure 5 shows an example input-output identification for this subsystem.

\section{Soft Manipulator}

The manipulator's dynamic model is symbolically parameterized by $\mathbf{N}$ masses $\mathbf{m}$, stiffnesses $\mathbf{k}$, and damping coefficients $\mathbf{b}$. In the actuated case, there are also $N$ additional actuator parameters, $N-1$ unknown coefficients $\mathbf{c}$ and a single time delay $t_{d}$. To reduce the parameter set $\mathbf{p}$ from $4 N$ parameters to $2 N+2$ parameters we make the following observations: according to the expression for $V_{\epsilon}$ in Section III-A.1 stiffness changes linearly with channel length $L$ and therefore we can replace $\mathbf{k}$ with $\frac{L_{i}}{L_{1}} k$ where $i$ is the segment
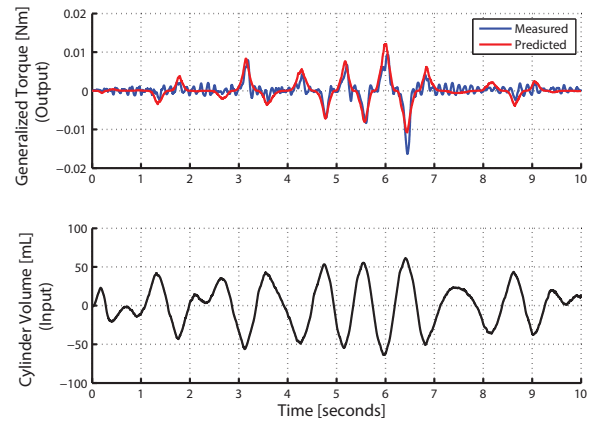

Fig. 5. Example experimental identification of a soft actuator subsystem. Again the identification process consists of exciting each independent subsystem with several randomized wave profiles, but here we fit and verify a two parameter nonlinear model to measured input-output data. Top: model predicted and measured output in blue and red respectively. Bottom: subsystem input.

index and $k$ is a single unknown stiffness. Furthermore, we hypothesize the non-conservative components of force $\mathbf{b} \dot{\theta}$ are similar along the length of the arm, therefore we approximate the coefficients $b_{i}$ to be equal $\forall i$.

\section{GRABBING EXPERIMENTS}

A primitive enabled by the developments in Sections III and IV is grabbing. Grabbing is defined as bringing the arm's end effector to a user specified, statically unreachable goal point with near zero tip velocity. Grabbing is an advantageous strategy to employ during manipulation as it enables the soft arm to reach areas that are statically unreachable due to gravity.

There are several major challenges that arise when trying to autonomously move the soft manipulator. First, we leave the top segment unactuated to accommodate external loads acting on the distal segments. Second, the system is tightly constrained by generalized torque limits. That is, the low operating pressures of the fluidic actuators in combination with their very low durometer rubber composition equate to constraints on input forces. To exemplify this problem consider the following search for feasible solutions that statically position the arm's end effector to a goal point in task space

$$
\begin{aligned}
& \text { find s.t. } \mathbf{C}-\mathbf{B} \boldsymbol{\tau}=\mathbf{0} \text {, }
\end{aligned}
$$

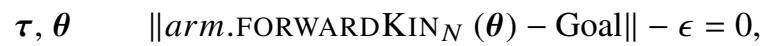

$$
\begin{aligned}
& \tau_{m}^{\min } \leq \tau_{m} \leq \tau_{m}^{\max } \quad \forall m=1 . . M, \\
& \theta_{n}^{\min } \leq \theta_{n} \leq \theta_{n}^{\max } \quad \text { and } \quad \dot{\theta}_{n}=0 \quad \forall n=1 . . N \text {. }
\end{aligned}
$$

By looking for solutions to goal points in the vicinity of the end effector, we quickly bring to light the limitations of a purely kinematic approach to motion planning for this class of manipulators subject to gravity. Table I depicts feasible static solutions in green for identified arms under estimated torque limits.

\section{A. Grabbing Algorithms}

We develop an algorithm, Algorithm 2, that can plan and execute a grab maneuver. The algorithm uses trajectory 
optimization to both plan a locally-optimal policy in generalized torque space as well as to determine an optimal input trajectory to the aggregate manipulation system to realize this policy. The trajectory optimizations were implemented using Drake [30]. Algorithm 2 can be interpreted as an iterative learning control, which after a couple grabbing attempts is able to successfully perform the desired maneuver. Here,

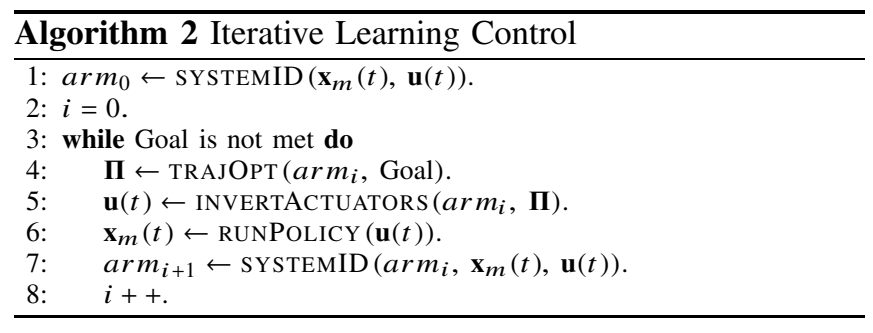

$\mathbf{x}_{m}(t)$ represents a measured state trajectory of the soft manipulator over the time interval $t=\left[0, t_{f}\right], \mathbf{u}(t)$ is the reference input trajectory to the manipulation system, and I represents a matrix of locally-optimal generalized torque and state trajectories. The function SYSTEMID describes the identification process in Section IV, the functions TRAJOPT and INVERTACTUATORS embody processes described in Subsections V-A.1 and V-A.2, and RUNPOLICY represents executing the reference input policy $\mathbf{u}(t)$ on the physical manipulation system.

1) Trajectory Optimization: We use a direct collocation approach to trajectory optimization [31] in line 4 of Algorithm 2. In short, this is a model-based open-loop policy search that finds a feasible input trajectory that moves the manipulator from an initial state to a goal state given both input and state constraints. The policy $\boldsymbol{\Pi}$ can generally be a function of both state and time, but in this case is parameterized by $M \times \frac{t_{f}}{\mathrm{~d} t}$ free parameters $\alpha$ where $M$ is the number of inputs and $\mathrm{d} t$ is a discrete time step

$$
\begin{aligned}
\boldsymbol{\Pi}_{\alpha}(\mathbf{x}, t) & =\alpha_{m, i} \quad \forall m=1 . . M, \\
i & =\left\lfloor\frac{t}{\mathrm{~d} t}\right\rfloor \quad \forall t=0 . . t_{f} .
\end{aligned}
$$

In the case of the soft manipulator each $\alpha$ is a generalized torque $\tau$ for each actuated segment augmented with the manipulator's state vector at each time step

$$
\boldsymbol{\Pi}_{\alpha}=\left[\begin{array}{lllll}
\boldsymbol{\tau}_{0} & \boldsymbol{\tau}_{1} & \boldsymbol{\tau}_{2} & \ldots & \boldsymbol{\tau}_{\frac{t_{f}}{\mathrm{~d} t}} \\
\mathbf{x}_{0} & \mathbf{x}_{1} & \mathbf{x}_{2} & \ldots & \mathbf{x}_{\frac{t_{f}}{\mathrm{~d} t}}
\end{array}\right] .
$$

The following trajectory optimization is performed to identify a locally-optimal policy $\boldsymbol{\Pi}_{\boldsymbol{\alpha}}^{*}$

$$
\begin{array}{ll}
\mathbf{\Pi}_{\alpha}^{*}=\min _{\alpha} & \sum_{i} g\left(\mathbf{x}_{i}, \boldsymbol{\tau}_{i}\right) \quad \Leftarrow \text { Objective Function } \\
\text { subject to } & 0=\mathbf{x}_{i}-f\left(\mathbf{x}_{i-1}, \boldsymbol{\tau}_{i-1}\right) \mathrm{d} t-\mathbf{x}_{0} \quad \forall i=1 \ldots \frac{t_{f}}{\mathrm{~d} t}, \\
& 0=h\left(\mathbf{x}_{\frac{t_{f}}{\mathrm{~d} t}}\right), \quad \Leftarrow \text { Enforce Tip Motion } \\
& \tau_{m}^{\min } \leq \tau_{m, i} \leq \tau_{m}^{\max } \text { and } \tau_{m, 0}=0 \quad \forall m, \forall i, \\
& \theta_{n}^{\min } \leq \theta_{n, i} \leq \theta_{n}^{\max } \forall n, \forall i, \\
& \theta_{n, 0} \leftarrow \text { measured } \quad \forall n d \dot{\theta}_{n, 0}=0 \quad \forall n .
\end{array}
$$

The first line of constraints forces the policy to obey the manipulator's dynamics and leverages a sequential quadratic program's ability to handle constraints. The second line consists of general nonlinear constraints enforced at the last point in the trajectory $t=t_{f}$. In the specific case of performing a $g r a b$ we formulate $h$ as follows:

$$
\begin{aligned}
& h_{p}=\| \text { arm.FORWARDKIN } N(\boldsymbol{\theta})-\operatorname{Goal} \|-\varepsilon_{p} \text {, } \\
& h_{v}=\| \text { arm.FORWARDVEL } N(\boldsymbol{\theta}, \dot{\boldsymbol{\theta}}) \|-\varepsilon_{v} \text {, }
\end{aligned}
$$

where $h_{p}$ constrains end effector position to the goal point and $h_{v}$ constrains end effector velocity to be near zero at the point in time the goal is reached. In both constraints $\varepsilon$ represents a definable error tolerance.

For the task of grabbing, the objective function $g()$ can be used to minimize end effector velocity at $t_{f}$, i.e. taking the form $g\left(\mathbf{x}_{\frac{t_{f}}{d t}}\right)=\|$ arm.FORWARDVEL $N\left(\mathbf{x}_{\frac{t_{f}}{d t}}\right) \|$. Alternatively, $g()$ can be used to find a minimal effort policy and take the form $g\left(\tau_{i}\right)=\tau_{i}^{\mathrm{T}} R \tau_{i}$, where $R$ is a scalar weight.

2) Inverting Actuators: The manipulator's motion is planned in reference to its generalized torques. Using the soft actuator model developed in Section IV-C, this motion plan can be expressed in reference to cylinder displacements $\mathbb{V}_{s}^{m}$, where superscript $m$ denotes an individual cylinder model for each input

$$
\mathbb{V}_{s}^{m}(t)= \begin{cases}-1^{2 / 3} \frac{\tau_{m}^{1 / 3}(t)}{a_{m}^{1 / 3}} & : \tau_{m}(t) \leq 0 \\ \frac{\tau_{m}^{1 / 3}(t)}{a_{m}^{1 / 3}} & : \tau_{m}(t)>0\end{cases}
$$

Since the target motion plan $\mathbb{V}_{s}^{*}(t)$ is a volume profile, many alternative drive systems can be used to realize the manipulator's trajectory. In this work we use fluidic drive cylinders and this approach allows us to closely match the prescribed volume profile. To effectively invert the LTI fluidic drive cylinder model, developed in the Section IVB, we use $M$ direct collocation trajectory optimizations. In these problems

$$
\boldsymbol{\Pi}_{\alpha}^{m}=\left[\begin{array}{ccccc}
u_{0}^{m} & u_{1}^{m} & u_{2}^{m} & \ldots & u_{t_{f}}^{m} \\
\mathbf{x}_{0}^{m} & \mathbf{x}_{1}^{m} & \mathbf{x}_{2}^{m} & \ldots & \mathbf{x}_{t_{f}}^{m} \\
& & & & \frac{\mathrm{d}}{\mathrm{d} t}
\end{array}\right]
$$

And the following optimization, performed for each cylinder model, identifies a locally-optimal reference input $\mathbf{u}^{*}(t)$. The superscript $m$ has been omitted for convenience

$$
\begin{array}{ll}
\mathbf{\Pi}_{\alpha}{ }^{*}=\min _{\alpha} & \sum_{i}\left\|\mathbb{V}_{s}(i)-\mathbf{C} \mathbf{x}_{i}+\mathbf{D} u_{i}\right\| \quad \Leftarrow \text { Track } \mathbb{V}_{s} \\
\text { subject to } & 0=\mathbf{x}_{i}-\left(\mathbf{A} \mathbf{x}_{i-1}+\mathbf{B} u_{i-1}\right) \mathrm{d} t-\mathbf{x}_{0} \quad \forall i=1 . . \frac{t_{f}}{\mathrm{~d} t}, \\
& u^{\min } \leq u_{i} \leq u^{\max } \quad \forall i \quad \text { and } \quad \mathbf{x}_{0}=0 .
\end{array}
$$

It is important to note that the locally-optimal input trajectories $\mathbf{u}^{*}(t)$ returned by the above optimization represent the best realization of a given volume profile subject to the dynamic limitations of the drive mechanism. For example, areas of high-frequency oscillation within $\tau^{*}(t)$ can result in significant localized tracking errors. As a solution, if the 
discrepancy between simulated model output and volume profile, i.e. $\left\|\mathbb{V}_{s}(t)-\mathbf{C} \mathbf{x}(t)+\mathbf{D} \mathbf{u}(t)\right\|$, exceeds an experimentally determined threshold for some span of time, we simply rerun the policy search procedure with a randomized $\tau(t)$ until a suitable realization is found. Alternative solutions may include planning directly in $\mathbf{u}$ space; however, this requires a single optimization to handle a dynamic model of the entire manipulation system, i.e. manipulator, actuator models, and cylinder models.

\section{B. Grabbing Evaluations}

In order to experimentally validate the outlined approach for grabbing with a soft and highly-compliant arm, we conduct multiple trials of four experiments, summarized in Table I. The goal of these experiments is to have the aggregate manipulation system autonomously perform a grab maneuver. A successful grab is defined as attaching to and removing a $4 \mathrm{~cm}$ diameter table tennis ball from a holder at the goal position; refer to Figure 1. Locally-optimal input trajectories $\mathbf{u}^{*}(t)$, as determined in Section V-A.2, are executed on the aggregate manipulation system. Trials reported in Table I and Figure 6 occurred after successful completion of Algorithm 2. The arm's torque limits are controlled and varied between experiments, i.e. experiments one and two to three and four. Among these groups goal location is also controlled for and varied, i.e. one to two and three to four. In experiments one and two the ball, represented as the black circle in Table I, is fixed at the user specified goal location around which the plan is derived. In experiments three and four the ball location underwent an initial one-time, experimentally determined adjustment by $2 \mathrm{~cm}$ to ensure it corresponded to the simulated realization of the plan, which considers the dynamic limitations of the fluidic drive system. Important simplifications: In these evaluations the unactuated regions between segments $\mathbf{L}_{p}$ were assumed zero. Additionally, for model stability purposes, the center of mass locations were redefined as

$\mathbf{P}_{n}=M_{\text {tip } n-1}^{\text {base }} \mathbf{R}_{z}(\gamma) \mathbf{T}_{z}\left(L_{P}\right) \mathbf{R}_{y}\left(\frac{\kappa s}{2}\right) \mathbf{T}_{z}\left(\frac{d(\kappa s)}{2}\right) \mathbf{0} \forall n$

This adjustment effectively amplifies center of mass motion as segment curvature increases; However, for segment curvatures achieved during these experiments, this model assumption captures the dynamics of interest.

TABLE I

SUMMARY OF GRABBING EXPERIMENTS

\begin{tabular}{|c|c|c|c|c|c|}
\hline $\begin{array}{c}\text { Exp. } \\
\#\end{array}$ & $\begin{array}{l}\text { Sys } \\
\text { IDs }\end{array}$ & $\begin{array}{l}\text { Consec. } \\
\text { Attempts }\end{array}$ & $\begin{array}{l}\text { Success } \\
\text { Grabs }\end{array}$ & \multicolumn{2}{|c|}{$\begin{array}{l}\text { Plan Realization } \\
\text { at } t=t_{f}\end{array}$} \\
\hline & & & t & 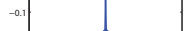 & . \\
\hline 3 & 2 & 5 & $4^{\star}$ & & \\
\hline 4 & 1 & 12 & $11^{\star}$ & 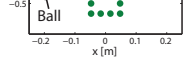 & 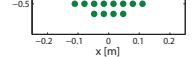 \\
\hline
\end{tabular}

The aggregate system was able to successfully grab the ball in approximately $92 \%$ of trials. Experiments one and two were performed consecutively. Although 2 iterations of system identification were performed on the actuator model parameter set during experiment one, no additional identifications were performed during experiment two. Similarly, experiments three and four were performed consecutively and two identifications were required during experiment three and one during experiment four.

Figure 6 shows the cartesian state trajectories of the manipulator's end effector for each experiment. Trials for which motion capture data was lost for a significant portion of time were omitted. This occurred when the end-effector endpoint was misinterpreted as the ball center-point and is a limitation of the experimental setup. Raw end effector velocity measurements were filtered using a 5-point moving average, removing jitter from numerical differencing.
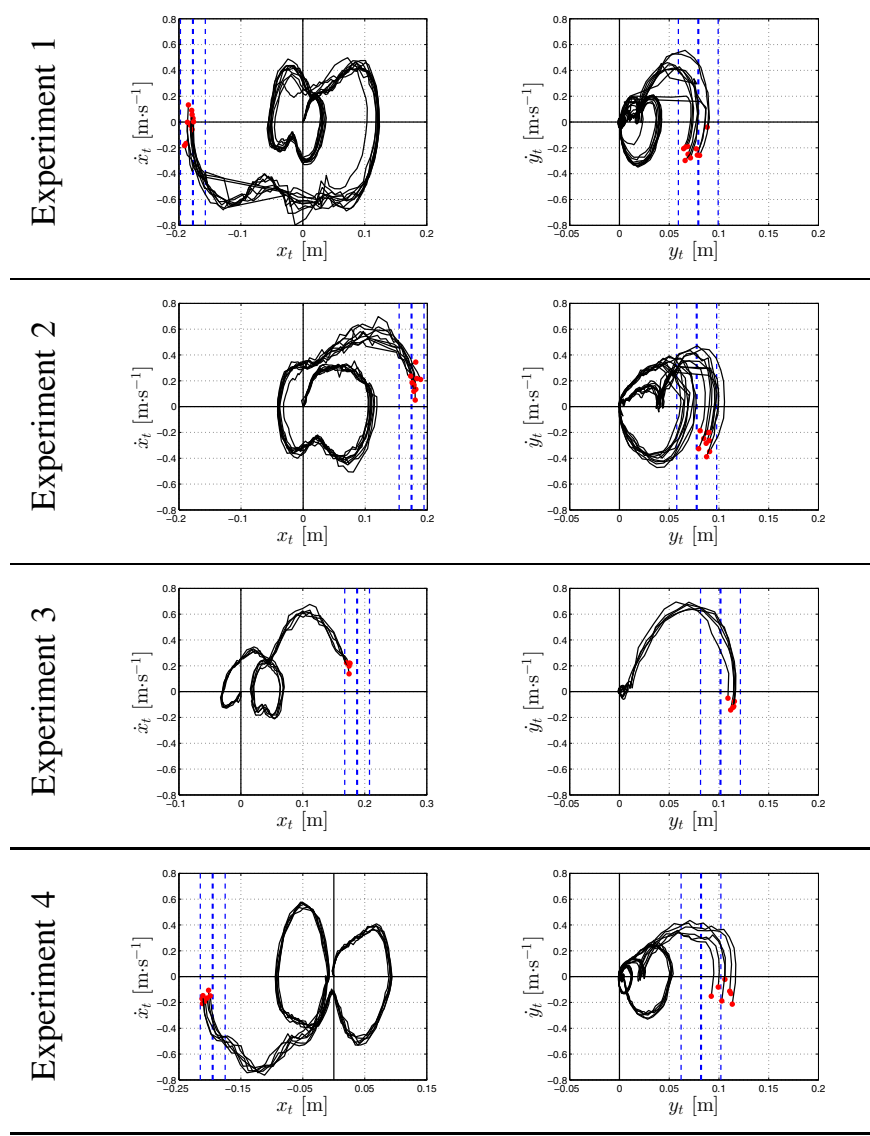

Fig. 6. Cartesian state trajectories of the manipulator's end effector for each experiment. The left and right figures show $x$ and $y$ tip velocity versus position, respectively. The trajectories of independent trials for each experiment are overlaid in black. These trajectories originate from the origin and terminate at red markers indicating $t=t_{f}$. The vertical blue lines represent planned end-effector realizations $\pm 2 \mathrm{~cm}$.

\section{CONCLUSION}

In these initial experiments we found it feasible to compute a sufficiently accurate dynamic model to make planning viable for a soft elastomer manipulator. However, to obtain the required performance for executing specific tasks, like 
grabbing, we found it necessary to use iterative learning control. Also, during grab experiments, hook and loop fasteners were used on the manipulator's end effector and the ball. To some degree, this mechanism compensated for positional errors as the ball and end effector were securely connected after the moment of contact. In future work, these trajectories may be stabilized using linear time-varying linear quadratic regulators (LTV LQRs) [32] making them robust to uncertainty in initial conditions and tolerant of modeling inaccuracies. Although this class of robot is well-suited for environmental contact (e.g. whole arm grasping and bracing), the modeling assumptions used here may not suffice under these conditions. This work suggests dynamic model-based planning and control may be an appropriate approach for soft robotics.

\section{ACKNOWLEDGMENTS}

The support by Robert Katzschmann, Jose Lara, and Jonathan Lambert is highly appreciated. This work was done in the Distributed Robotics Laboratory at MIT with support from the National Science Foundation, grant numbers NSF 1117178, NSF EAGER 1133224, NSF IIS1226883, and NSF CCF1138967 as well as NSF GRFP, primary award number 1122374.

\section{REFERENCES}

[1] G. Robinson and J. B. C. Davies, "Continuum robots-a state of the art," in Robotics and Automation, 1999. Proceedings. 1999 IEEE International Conference on, vol. 4. IEEE, 1999, pp. 2849-2854.

[2] M. W. Hannan and I. D. Walker, "Kinematics and the implementation of an elephant's trunk manipulator and other continuum style robots," Journal of Robotic Systems, vol. 20, no. 2, pp. 45-63, 2003.

[3] B. A. Jones and I. D. Walker, "Practical kinematics for real-time implementation of continuum robots," Robotics, IEEE Transactions on, vol. 22, no. 6, pp. 1087-1099, 2006.

[4] B. A. Jones and I. D. Walker, "Kinematics for multisection continuum robots," Robotics, IEEE Transactions on, vol. 22, no. 1, pp. 43-55, 2006.

[5] G. S. Chirikjian and J. W. Burdick, "Kinematically optimal hyperredundant manipulator configurations," Robotics and Automation, IEEE Transactions on, vol. 11, no. 6, pp. 794-806, 1995.

[6] J. Xiao and R. Vatcha, "Real-time adaptive motion planning for a continuum manipulator," in Intelligent Robots and Systems (IROS), 2010 IEEE/RSJ International Conference on. IEEE, 2010, pp. 59195926.

[7] G. S. Chirikjian, "Hyper-redundant manipulator dynamics: a continuum approximation," Advanced Robotics, vol. 9, no. 3, pp. 217-243, 1994.

[8] I. A. Gravagne and I. D. Walker, "Uniform regulation of a multisection continuum manipulator," in Robotics and Automation, 2002. Proceedings. ICRA'02. IEEE International Conference on, vol. 2. IEEE, 2002, pp. 1519-1524.

[9] I. A. Gravagne, C. D. Rahn, and I. D. Walker, "Large deflection dynamics and control for planar continuum robots," Mechatronics, IEEE/ASME Transactions on, vol. 8, no. 2, pp. 299-307, 2003.

[10] J. Snyder and J. Wilson, "Dynamics of the elastica with end mass and follower loading," Journal of applied mechanics, vol. 57, no. 1, pp. 203-208, 1990.

[11] J. Wilson and J. Snyder, "The elastica with end-load flip-over," Journal of applied mechanics, vol. 55, no. 4, pp. 845-848, 1988.

[12] E. Tatlicioglu, I. D. Walker, and D. M. Dawson, "Dynamic modelling for planar extensible continuum robot manipulators," in Robotics and Automation, 2007 IEEE International Conference on. IEEE, 2007, pp. 1357-1362.

[13] D. Braganza, D. M. Dawson, I. D. Walker, and N. Nath, "A neural network controller for continuum robots," Robotics, IEEE Transactions on, vol. 23, no. 6, pp. 1270-1277, 2007.
[14] W. McMahan, B. A. Jones, and I. D. Walker, "Design and implementation of a multi-section continuum robot: Air-octor," in Intelligent Robots and Systems, 2005.(IROS 2005). 2005 IEEE/RSJ International Conference on. IEEE, 2005, pp. 2578-2585.

[15] R. F. Shepherd, F. Ilievski, W. Choi, S. A. Morin, A. A. Stokes, A. D. Mazzeo, X. Chen, M. Wang, and G. M. Whitesides, "Multigait soft robot," Proceedings of the National Academy of Sciences, vol. 108, no. 51, pp. 20400-20 403, 2011.

[16] C. D. Onal, X. Chen, G. M. Whitesides, and D. Rus, "Soft mobile robots with on-board chemical pressure generation," in International Symposium on Robotics Research (ISRR), 2011.

[17] A. D. Marchese, C. D. Onal, and D. Rus, "Soft robot actuators using energy-efficient valves controlled by electropermanent magnets," in Intelligent Robots and Systems (IROS), 2011 IEEE/RSJ International Conference on. IEEE, 2011, pp. 756-761.

[18] C. D. Onal and D. Rus, "Autonomous undulatory serpentine locomotion utilizing body dynamics of a fluidic soft robot," Bioinspiration \& biomimetics, vol. 8, no. 2, p. 026003, 2013.

[19] A. D. Marchese, C. D. Onal, and D. Rus, "Autonomous soft robotic fish capable of escape maneuvers using fluidic elastomer actuators," Soft Robotics, vol. 1, no. 1, pp. 75-87, 2014.

[20] A. D. Marchese, K. Komorowski, C. D. Onal, and D. Rus, "Design and control of a soft and continuously deformable $2 \mathrm{~d}$ robotic manipulation system," in Robotics and Automation (ICRA), 2014 IEEE International Conference on. IEEE, 2014, pp. 2189-2196.

[21] A. D. Marchese, R. K. Katzschmann, and D. Rus, "Whole arm planning for a soft and highly compliant $2 \mathrm{~d}$ robotic manipulator," in Intelligent Robots and Systems (IROS), 2014 IEEE/RSJ International Conference on. IEEE, 2014, pp. 554-560.

[22] M. Calisti, A. Arienti, M. Giannaccini, M. Follador, M. Giorelli, M. Cianchetti, B. Mazzolai, C. Laschi, and P. Dario, "Study and fabrication of bioinspired octopus arm mockups tested on a multipurpose platform," in Biomedical Robotics and Biomechatronics (BioRob), 2010 3rd IEEE RAS and EMBS International Conference on. IEEE, 2010, pp. 461-466.

[23] C. Laschi, M. Cianchetti, B. Mazzolai, L. Margheri, M. Follador, and P. Dario, "Soft robot arm inspired by the octopus," Advanced Robotics, vol. 26, no. 7, pp. 709-727, 2012.

[24] M. Calisti, M. Giorelli, G. Levy, B. Mazzolai, B. Hochner, C. Laschi, and P. Dario, "An octopus-bioinspired solution to movement and manipulation for soft robots," Bioinspiration \& biomimetics, vol. 6, no. 3, p. 036002, 2011.

[25] T. Umedachi, V. Vikas, and B. Trimmer, "Highly deformable 3-d printed soft robot generating inching and crawling locomotions with variable friction legs," in Intelligent Robots and Systems (IROS), 2013 IEEE/RSJ International Conference on, Nov 2013, pp. 4590-4595.

[26] M. Luo, M. Agheli, and C. D. Onal, "Theoretical modeling and experimental analysis of a pressure-operated soft robotic snake," Soft Robotics, vol. 1, no. 2, pp. 136-146, 2014.

[27] R. V. Martinez, J. L. Branch, C. R. Fish, L. Jin, R. F. Shepherd, R. Nunes, Z. Suo, and G. M. Whitesides, "Robotic tentacles with three-dimensional mobility based on flexible elastomers," Advanced Materials, vol. 25, no. 2, pp. 205-212, 2013.

[28] A. D. Marchese and D. Rus, "Design, kinematics, and control of a soft spatial fluidic elastomer manipulator," 2014, manuscript submitted for publication.

[29] R. J. Webster and B. A. Jones, "Design and kinematic modeling of constant curvature continuum robots: A review," The International Journal of Robotics Research, vol. 29, no. 13, pp. 1661-1683, 2010.

[30] R. Tedrake, "Drake: A planning, control, and analysis toolbox for nonlinear dynamical systems," 2014. [Online]. Available: http://drake.mit.edu

[31] O. von Stryk, "Numerical solution of optimal control problems by direct collocation," in Optimal Control, ser. ISNM International Series of Numerical Mathematics, R. Bulirsch, A. Miele, J. Stoer, and K. Well, Eds. Birkhuser Basel, 1993, vol. 111, pp. 129-143.

[32] R. Tedrake, "LQR-trees: Feedback motion planning on sparse randomized trees," in Proceedings of Robotics: Science and Systems, Seattle, USA, June 2009. 